\title{
A REVIEW OF AN OPTIMAL DESIGN PROBLEM FOR A PLATE OF VARIABLE THICKNESS*
}

\author{
JULIO MUÑOZ ${ }^{\dagger}$ AND PABLO PEDREGAL ${ }^{\ddagger}$
}

\begin{abstract}
We revisit a classic design problem for a plate of variable thickness under the model of Kirchhoff. Our main contribution has two goals. One is to provide a rather general existence result under a main assumption on the structure of the tensor of material constants. The other focuses on providing a minimal number of additional design variables for a relaxation of the problem when that assumption on the tensor of elastic constants does not hold. In both situations, the cost functional can be pretty general.
\end{abstract}

Key words. optimal design, direct method, existence, relaxation

AMS subject classifications. 49J25, 49J20, 35J50, 74P04

DOI. $10.1137 / 050639569$

1. Introduction. The problem of the optimal design of a plate of variable thickness under Kirchhoff's model can be stated as finding the optimal, symmetric profile

$$
h: \Omega \subset \mathbf{R}^{2} \rightarrow \mathbf{R},
$$

where $\Omega$ is supposed to be the midplane with respect to which the plate is symmetric, so that it minimizes the value of the compliance functional

$$
I(h)=\int_{\Omega} f(x) u(x) d x,
$$

where $f$ is the vertical load over the plate, and $u$ is the vertical displacement in equilibrium which is obtained from the profile $h$ by solving the equation of equilibrium

$$
\sum_{i, j, k, l} \frac{\partial^{2}}{\partial x_{i} \partial x_{j}}\left(h^{3}(x) M_{i j k l} \frac{\partial^{2} u(x)}{\partial x_{k} \partial x_{l}}\right)=f(x)
$$

in $\Omega$, supplemented with clamped boundary conditions around $\partial \Omega$ by demanding $u=\nabla u=0$ over $\partial \Omega$. Here the fourth-order tensor $M$ encloses the various material constants for the type of elastic material the plate is made of. In addition, there should be some other constraints on the admissible profiles so that the problem is meaningful. On the one hand, we assume that there is a minimum and a maximum height for $h$ so that

$$
0<h_{-} \leq h(x) \leq h_{+}
$$

*Received by the editors September 5, 2005; accepted for publication (in revised form) August 12, 2006; published electronically February 23, 2007. This work was supported by project MTM200407114 from Ministerio de Educación y Ciencia (Spain).

http://www.siam.org/journals/sicon/46-1/63956.html

${ }^{\dagger}$ Departamento de Matemáticas, Facultad de Medio Ambiente, Universidad de Castilla-La Mancha, 071 Toledo, Spain (julio.munoz@uclm.es). The work of this author was supported by project PBC-05-010-1 from JCCM (Castilla-La Mancha).

¥Departamento de Matemáticas, ETSI Industriales, Universidad de Castilla-La Mancha, 13071 Ciudad Real, Spain (pablo.pedregal@uclm.es). The work of this author was supported by project PAI05-029 from JCCM (Castilla-La Mancha). 
and $h_{-}, h_{+}$are given parameters. On the other hand, we must limit the amount of material that can be used so that

$$
\int_{\Omega} h(x) d x \leq V|\Omega|
$$

and $h_{-}<V<h_{+}$.

This problem has received some attention over the years in two different directions. First, it was noticed long ago that, at least in some situations, this problem is not wellposed in the sense that there might not exist optimal profiles (see [6], [7]). Today, this is a well-understood fact. It is typically associated with some lack of convexity, often taken in a suitable broad sense. This direction was further pursued and explored from the horizon of finding a minimal relaxation in the sense of using a minimal number of generalized design variables (see [5], [9]). Several later works emphasized this perspective and proved various types of results always trying to minimize in various ways the number of design variables needed to describe minimizing profiles. In many of these contributions, Young measures associated with minimizing profiles were used in one way or another (see [1], [3], [4], [11], [15]). Second, in some other situations, existence of optimal profiles has been shown despite the fact of the just-mentioned difficulties (see [14], [16], [17]), coming to a situation where it is not completely clear when, depending on the ingredients, one can trust existence results or else anticipate highly oscillating optimal profiles. Another point in many of these works is that the only cost functional considered is the compliance written before, along with some other variants of order zero (no derivatives of $u$ ).

The aim of our contribution here is twofold. First, we examine the structural ingredients of the problem that enable an existence result, and how existence of optimal profiles is compromised when such requirements are not fulfilled. As we will see, this is basically related to the structure of the tensor $M$ of material constants so that the existence of optimal profiles for this problem depends (for many relevant cost functionals) on the elasticity properties of the material we use to manufacture the plate. Second, we would like to be able to examine more general cost functionals and not just the compliance. We will give results for much more general objective functionals in both existence as well as nonexistence cases.

Let $F(x, u, \lambda, \xi, h): \Omega \times \mathbf{R} \times \mathbf{R}^{2} \times \mathbf{M}^{2 \times 2} \times \mathbf{R} \rightarrow \mathbf{R}$ be a given integrand, continuous in the variables $(u, \lambda, \xi, h)$ and measurable in $x$ (here $\mathbf{M}^{2 \times 2}$ is the space of the $2 \times 2$ real matrices). Define

$$
I(h)=\int_{\Omega} F\left(x, u(x), \nabla u(x), \nabla^{2} u(x), h(x)\right) d x,
$$

where $u$ solves

$$
\begin{aligned}
\sum_{i, j, k, l} \frac{\partial^{2}}{\partial x_{i} \partial x_{j}}\left(h^{3}(x) M_{i j k l} \frac{\partial^{2} u(x)}{\partial x_{k} \partial x_{l}}\right) & =f(x) \text { in } \Omega, \\
u(x) & =\frac{\partial u(x)}{\partial n}=0 \text { on } \partial \Omega,
\end{aligned}
$$

Specifically, we consider the following optimal design problem:

$$
\begin{aligned}
\text { Minimize } & I(h) \\
\text { subject to } & h_{-} \leq h(x) \leq h_{+} \text {in } \Omega, \quad \int_{\Omega} h(x) d x \leq V|\Omega| .
\end{aligned}
$$


The main structural assumption to distinguish between existence and nonexistence of optimal solutions for this optimal design problem refers to the material tensor $M$. We will say that $M$ is decomposable if

$$
M=M_{1} \otimes M_{2},
$$

where $M_{i}$ are positive definite, second-order tensors (matrices). Notice how in this case the equilibrium equation basically reduces to the biharmonic operator. In this situation, we have a general existence result.

TheOREM 1. Suppose that $M=M_{1} \otimes M_{2}$, i.e., $M$ is decomposable, and the integrand $F$ in the cost functional $I$ is such that the functions

$$
\xi \in\left\{\mathbf{M}^{2 \times 2}: \frac{c}{M_{2} \cdot \xi}>0\right\} \mapsto F\left(x, u, \lambda, \xi, \frac{c}{\left(M_{2} \cdot \xi\right)^{1 / 3}}\right)
$$

and

$$
(\xi, z) \in\left\{\mathbf{M}^{2 \times 2}: M_{2} \cdot \xi=0\right\} \times \mathbf{R} \mapsto \min _{h \in\left[h_{-}, z\right] \cap Q} F(x, u, \lambda, \xi, h)
$$

are convex for any constant $c$ and fixed $(x, u, \lambda)$. Then there are optimal profiles for the associated optimal design problem for the plate.

A corollary worth stating covers many situations of interest.

Corollary 2. Suppose that the integrand $F$ does not depend on $\xi$ and $h$, and $M$ is decomposable. Then for any such $F$ (even nonconvex), the corresponding optimal design problem admits optimal solutions.

Explicit cases like the compliance $F=f(x) u(x)$ are covered with this corollary. But also examples like $F=g(x) u(x), F=|\nabla u(x)|^{2}$, etc., can be treated through this result as well.

When the tensor $M$ is not decomposable, the situation is drastically different. In many cases, this fact is responsible for the lack of optimal solutions and the analysis is much more complex. See the references cited above. In the particular situation where we assume that the profile $h$ is a function of $x_{1}$ alone, so that $h(x)=h\left(x_{1}\right)$, and the tensor $M$ is that of an orthotropic material, a relaxed formulation of the problem can be pursued. It has been a principal goal over the years to find a minimal full relaxation of this problem, that is, one which requires the least number of additional design variables. For the compliance functional, the best result we know of has been obtained in [4] (also in [15] within a more general framework). Here, by revisiting some of our own old ideas [11], we are able to show that this same result holds true even for much more general functionals. Recall that $M$ for orthotropic materials is defined in terms of two main material parameters: Young's modulus E, and Poisson's ratio $r$, so that the nonvanishing components of $M$ are

$$
\begin{aligned}
& M_{1111}=M_{2222}=\frac{2}{3} \frac{E}{1-r^{2}}, \quad M_{1122}=M_{2211}=\frac{2}{3} \frac{E r}{1-r^{2}}, \\
& M_{1212}=M_{1221}=M_{2112}=M_{2121}=\frac{1}{3} \frac{E}{1+r} .
\end{aligned}
$$

TheOREM 3. Let admissible profiles depend only on $x_{1}, M$ corresponding to an orthotropic material, and let the integrand for the cost functional

$$
F(x, u, \lambda): \Omega \times \mathbf{R} \times \mathbf{R}^{2} \rightarrow \mathbf{R}
$$


be measurable in $x \in \Omega$ and continuous (not necessarily convex) in the pairs $(u, \lambda)$. Consider the optimal design problem

$$
\text { Minimize in }(\theta, h): \quad J(\theta, h)=\int_{\Omega} F(x, u(x), \nabla u(x)) d x
$$

subject to

$$
\begin{aligned}
\theta & \in[0,1], \quad h \in\left[h_{-}, h_{+}\right], \\
\int_{\Omega}\left[\theta(x) h_{+}+(1-\theta(x)) h(x)\right] d x & \leq V|\Omega|,
\end{aligned}
$$

and where $u$ solves

$$
\begin{aligned}
\sum_{i, j, k, l} \frac{\partial^{2}}{\partial x_{i} \partial x_{j}}\left(\bar{M}_{i j k l} \frac{\partial^{2} u}{\partial x_{k} \partial x_{l}}\right) & =f \text { in } \Omega, \\
u & =\frac{\partial u}{\partial n}=0 \text { on } \partial \Omega,
\end{aligned}
$$

and the nonvanishing components of $\bar{M}$ depend on the design variables through formulae

$$
\begin{aligned}
\bar{M}_{1111} & =\frac{2}{3} c(x) \frac{E}{1-r^{2}}, \quad \bar{M}_{2222}=\frac{2}{3} m(x) E+\frac{2}{3} c(x) \frac{E r^{2}}{1-r^{2}}, \\
\bar{M}_{1122} & =\bar{M}_{2211}=\frac{2}{3} c(x) \frac{E r}{1-r^{2}}, \\
\bar{M}_{1212} & =\bar{M}_{1221}=\bar{M}_{2112}=\bar{M}_{2121}=\frac{1}{3} m(x) \frac{E}{1+r}, \\
m(x) & =\theta(x) h_{+}^{3}+(1-\theta(x)) h^{3}(x), \\
c(x)^{-1} & =\theta(x) h_{+}^{-3}+(1-\theta(x)) h(x)^{-3} .
\end{aligned}
$$

This problem is a full relaxation of the initial optimal design problem in the sense

$$
\inf _{h} I(h)=\min _{(\theta, h)} J(\theta, h) .
$$

The relevance of this result is in the fact that only one additional design variable, $\theta$ (a certain weight), is required to produce a full relaxation of the problem, and this is so for a rather huge class of cost functionals and not only for the compliance. We will later provide further details as to how one is to interpret these pairs $(\theta, h)$ in terms of sequences of profiles for the original problem.

This work includes another three sections. The second one contains the full proof of Theorem 1 as well as some observations on some explicit examples. Section 3 covers a brief, elementary discussion on the structure of the material tensor $M$. Finally, the last section is devoted to the proof of Theorem 3. We will also dwell on the interpretation of the proposed relaxed formulation in terms of the ingredients of the original optimal design problem.

2. Existence results. It is our aim to study a type of design problem for plates whose state equation has the format

$$
\operatorname{div}\left(\operatorname{div}\left(h^{3}(x)\left(M_{1} \otimes M_{2}\right) \nabla^{2} u(x)\right)\right)=f(x) \text { in } \Omega .
$$


It is assumed that $f \in L^{2}(\Omega)$ is the applied vertical load, $\Omega$ is a smooth bounded domain in $\mathbf{R}^{2}$ that represents the midplane of the plate, $h \in L^{\infty}(\Omega)$ is the design variable, and the tensors $M_{i}$ are assumed to be positive and symmetric. To this equation we add the boundary conditions

$$
u(x)=\frac{\partial u(x)}{\partial n}=0 \text { on } \partial \Omega
$$

(clamped plate). We assume further natural constraints on the feasible designs by limiting the height of the thicknesses and the amount of material: the set $\mathcal{H}$ of admissible designs is defined as

$$
\text { (3) } \mathcal{H}=\left\{h \in L^{\infty}(\Omega): h(x) \in\left[h_{-}, h_{+}\right] \doteq Q \text { a.e. } x \in \Omega, V(h) \doteq \int_{\Omega} h(x) d x \leq V\right\}
$$

( $V, 0<h_{-}<h_{+}$are given positive constants). Associated with this state equation, we consider the general optimization problem

$$
\min _{h \in \mathcal{H}}\left\{L(h) \doteq \int_{\Omega} F\left(x, u, \nabla u(x), \nabla^{2} u(x), h(x)\right) d x\right\},
$$

where $u$ solves (1)-(2) and $F$ is a given integrand such that

$$
F:(x, u, \lambda, \xi, h) \in \Omega \times \mathbf{R} \times \mathbf{R}^{2} \times \mathbf{M}^{2 \times 2} \times Q \rightarrow \overline{\mathbf{R}}=\mathbf{R} \cup\{+\infty\} .
$$

$F$ is measurable in $x$ and continuous in $(u, \lambda, \xi, h)$.

Our goal is the optimization problem that consists of looking for an admissible $h$ and the corresponding displacement $u$, the only weak solution of problem (1)-(2) in the Sobolev space $H_{0}^{2}(\Omega)$ (the subspace of $H^{2}(\Omega)$ under the constraints (2)), which minimizes the objective functional $L$ defined in $(4)$. We denote this problem by $(\mathcal{P})$.

For the study of the above minimization problem, we shall consider a new equivalent variational problem. The underlying idea is to use the differential expression (1) in order to define a new objective functional subject to a set of constraints which are easier to deal with. The construction of this equivalent problem is performed in an elementary way [13]:

1. We introduce an auxiliary function $u_{0}$ : the solution of the elliptic problem

$$
-\operatorname{div}\left(M_{1} \nabla u_{0}\right)=f, \quad u_{0} \in H_{0}^{1}(\Omega) .
$$

2. Equations (1) and (5) give

$$
\operatorname{div} \operatorname{div}\left(h^{3}\left(M_{1} \otimes M_{2}\right) \nabla^{2} u\right)+\operatorname{div}\left(M_{1} \nabla u_{0}\right)=0,
$$

which is equivalent to writing

$$
\operatorname{div}\left(M_{1} \nabla\left(h^{3}\left[\operatorname{div}\left(M_{2} \nabla u\right)\right]\right)+M_{1} \nabla u_{0}\right)=0
$$

or even

$$
\operatorname{div}\left(M_{1} \nabla\left(h^{3}\left[M_{2} \cdot \nabla^{2} u\right]\right)+M_{1} \nabla u_{0}\right)=0,
$$

i.e.,

$$
\operatorname{div}\left(M_{1} \nabla v\right)=0,
$$

where

$$
v=h^{3} \operatorname{div}\left(M_{2} \nabla u\right)+u_{0}=h^{3} M_{2} \cdot \nabla^{2} u+u_{0} .
$$


3. The new optimization problem, denoted by $(\mathcal{E P})$, is described as follows. The new integrand for the cost functional is

$$
\varphi(x, u, \lambda, \xi, v, z)=\min _{\widetilde{h} \in Q}\left\{F(x, u, \lambda, \xi, \widetilde{h}): v=\widetilde{h}^{3}\left(M_{2} \cdot \xi\right)+u_{0}(x), z \geq \widetilde{h}\right\}
$$

understood as taking the value $+\infty$ whenever the set of admissible $h$ 's is empty, and the objective functional to be minimized in the variables $(u, v, z)$ is

$$
J(u, v, z)=\int_{\Omega} \varphi\left(x, u(x), \nabla u(x), \nabla^{2} u(x), v(x), z(x)\right) d x,
$$

under the constraints

$$
u \in H_{0}^{2}(\Omega), v \in H^{1}(\Omega), \operatorname{div}\left(M_{1} \nabla v\right)=0, z \in L^{\infty}(\Omega), \int_{\Omega} z(x) d x=V .
$$

Proposition 4. The two optimization problems $(\mathcal{P})$ and $(\mathcal{E} \mathcal{P})$ are equivalent in the following sense: for any admissible pair $(h, u)^{1}$ for $(\mathcal{P})$ there is a triplet $(u, v, z)$ admissible for $(\mathcal{E P})$ such that

$$
L(h) \geq J(u, v, z)
$$

Conversely, for any admissible triplet $(u, v, z)$ for $(\mathcal{E P})$, there is an admissible pair $(h, u)$ for $(\mathcal{P})$ and

$$
L(h)=J(u, v, z)
$$

In particular, if $(u, v, z)$ is optimal for $(\mathcal{E P})$, then

$$
h(x)=\left(\frac{v(x)-u_{0}(x)}{M_{2} \cdot \nabla^{2} u(x)}\right)^{\frac{1}{3}}
$$

whenever $M_{2} \cdot \nabla^{2} u(x) \neq 0$, and

$$
h(x)=\arg \min _{\widetilde{h} \in Q}\left\{F\left(x, u(x), \nabla u(x), \nabla^{2} u(x), \widetilde{h}\right): z(x) \geq \widetilde{h}\right\}
$$

otherwise, is an optimal profile for $(\mathcal{P})$.

Proof. The proof is almost straightforward. We include some details for the convenience of the reader.

Let $(h, u)$ be admissible for $(\mathcal{P})$, so that problem $(1)-(2)$ holds. We consider $u_{0}$ (solution of $(5))$, and

$$
v(x)=h^{3}(x)\left(M_{2} \cdot \nabla^{2} u(x)\right)+u_{0}(x) .
$$

By following the construction explained above $v$ solves (8) and the classical regularity results on elliptic systems ensure that $v$ is in $H^{2}(\Omega)$. We select $z$ verifying

$$
z(x) \geq h(x), \quad z(x) \in Q, \quad \text { and } \quad \int_{\Omega} z(x) d x=V .
$$

\footnotetext{
${ }^{1}$ Here $(h, u)$ is said to be admissible in the sense that for any $h \in \mathcal{H}$ we find the only solution $u$ of problem (1)-(2).
} 
Then for any $x \in \Omega$,

$$
\begin{aligned}
& \varphi\left(x, u(x), \nabla u(x), \nabla^{2} u(x), v(x), z(x)\right) \\
= & \min _{\widetilde{h} \in Q}\left\{F\left(x, u, \nabla u(x), \nabla^{2} u(x), \widetilde{h}\right): v(x)=\widetilde{h}^{3}\left(M_{2} \cdot \nabla^{2} u(x)\right)+u_{0}(x), z(x) \geq \widetilde{h}\right\} \\
\leq & F\left(x, u, \nabla u(x), \nabla^{2} u(x), h(x)\right) .
\end{aligned}
$$

It is clear that our triplet, $(u, v, z)$, is admissible for $(\mathcal{E P})$ and $J(u, v, z) \leq L(h)$.

Let $(u, v, z)$ now be admissible for $(\mathcal{E P})$. The multifunction $H$ given by

$\arg \min _{\widetilde{h} \in Q}\left\{F\left(x, u(x), \nabla u(x), \nabla^{2} u(x), \widetilde{h}\right): v(x)=\widetilde{h}^{3}\left(M_{2} \cdot \nabla^{2} u(x)\right)+u_{0}(x) z(x) \geq \widetilde{h}\right\}$

is measurable and takes closed set values. Then $H$ admits a measurable selection (see [10, Thm. 2.23]) and we can select a measurable function $h$ such that $h(x) \in Q$, and for any $x \in \Omega$

$$
\begin{aligned}
& \varphi\left(x, u(x), \nabla u(x), \nabla^{2} u(x), v(x), z(x)\right) \\
= & \min _{\widetilde{h} \in Q}\left\{F\left(x, u(x), \nabla u(x), \nabla^{2} u(x), \widetilde{h}\right): v(x)=\widetilde{h}^{3}\left(M_{2} \cdot \nabla^{2} u(x)\right)+u_{0}(x), z(x) \geq \widetilde{h}\right\} \\
= & F\left(x, u(x), \nabla u(x), \nabla^{2} u(x), h(x)\right) .
\end{aligned}
$$

Moreover, by definition we have $v(x)=h^{3}(x)\left(M_{2} \cdot \nabla^{2} u(x)\right)+u_{0}(x), z(x) \geq h(x) \in$ $Q$. This is enough to fulfill the state equation

$$
\operatorname{div} \operatorname{div}\left(h^{3}\left(M_{1} \otimes M_{2}\right) \nabla^{2} u\right)=f(x),
$$

the bound on the volume

$$
\int_{\Omega} h(x) d x \leq \int_{\Omega} z(x) d x=V,
$$

and the equality $I(u, v, z)=L(h, u)$.

We can now establish the existence of optimal solutions for $(\mathcal{E P})$.

TheOREM 5. Assume that the two functions

$$
\xi \in\left\{\mathbf{M}^{2 \times 2}: \frac{c}{M_{2} \cdot \xi}>0\right\} \mapsto F\left(x, u, \lambda, \xi, \frac{c}{\left(M_{2} \cdot \xi\right)^{1 / 3}}\right)
$$

and

$$
(\xi, z) \in\left\{\mathbf{M}^{2 \times 2}: M_{2} \cdot \xi=0\right\} \times \mathbf{R} \mapsto \min _{h \in\left[h_{-}, z\right] \cap Q} F(x, u, \lambda, \xi, h)
$$

are convex for any constant $c$ and fixed $(x, u, \lambda)$. Problem $(\mathcal{E P})$,

$$
\inf _{(u, v, z)} J(u, v, z) \doteq \int_{\Omega} \varphi\left(x, u(x), \nabla u(x), \nabla^{2} u(x), v(x), z(x)\right) d x,
$$

where

$$
\varphi(x, u, \lambda, \xi, v, z)=\min _{h \in Q}\left\{F(x, u, \lambda, \xi, h): v=h^{3}\left(M_{2} \cdot \xi\right)+u_{0}(x), z \geq h\right\}
$$


under the restrictions

$$
u \in H_{0}^{2}(\Omega), v \in H^{1}(\Omega), \operatorname{div}\left(M_{1} \nabla v\right)=0, z \in L^{\infty}(\Omega), \int_{\Omega} z(x) d x=V,
$$

has optimal solutions.

Proof. Let $\left(u_{j}, v_{j}, z_{j}\right)$ be a minimizing sequence for $(\mathcal{E P})$. As we have seen in the proof of Proposition 4 we can build the corresponding sequence $h_{j}$ such that

$$
\operatorname{div} \operatorname{div}\left(h_{j}^{3}\left(M_{1} \otimes M_{2}\right) \nabla^{2} u_{j}\right)=f(x), u_{j} \in H_{0}^{2}(\Omega) .
$$

Then we can ensure that $u_{j}$ is uniformly bounded in $H^{2}(\Omega)$. This sequence converges to $u$ weakly in $H_{0}^{2}(\Omega)$ and, consequently, $u_{j}$ and $\nabla u_{j}$ converge strongly in $L^{2}(\Omega)$ to $u$ and $\nabla u$, respectively. On the other hand, by elliptic theory, $\operatorname{div}\left(M_{1} \nabla v_{j}\right)=0$ implies $v_{j}$ converges almost everywhere to a function $v \in H^{1}(\Omega)$ verifying the same elliptic equation (see [16] for a very neat proof in the case of the Laplacian), so that $v_{j}$ converges strongly to $v$. Finally, notice that because $\varphi(x, u, \lambda, \xi, v, z)=$ $\varphi\left(x, u, \lambda, \xi, v, h_{+}\right)$if $z_{j} \geq h_{+}$, we may assume without lost of generality that $h_{j} \leq$ $z_{j} \leq h_{+}$. Then $z_{j}$ converges to some $z$ in $L^{\infty}(\Omega)$ weak- $\star$, and this limit must satisfy $\int_{\Omega} z(x) d x=V$.

On the basis of these remarks, it remains to prove that $\varphi$ is jointly convex in $(\xi, z)$ for fixed $(x, u, \lambda, v)$. To do that, it is enlightening to rewrite $\varphi$ as

$$
\begin{cases}F\left(x, u, \lambda, \xi,\left(\frac{v-u_{0}(x)}{M_{2} \cdot \xi}\right)^{1 / 3}\right), & M_{2} \cdot \xi \neq 0, z \geq\left(\frac{v-u_{0}(x)}{M_{2} \cdot \xi}\right)^{1 / 3} \in Q \\ \min _{h \in\left[h_{-}, z\right] \cap Q} F(x, u, \lambda, \xi, h), & M_{2} \cdot \xi=0, v=u_{0}(x) \\ +\infty & \text { else }\end{cases}
$$

and discuss the convexity by considering two main cases:

1. $v \neq u_{0}(x)$ : in this case $\varphi$ is given by

$$
\begin{cases}F\left(x, u, \lambda, \xi,\left(\frac{v-u_{0}(x)}{M_{2} \cdot \xi}\right)^{1 / 3}\right), & M_{2} \cdot \xi \neq 0, z \geq\left(\frac{v-u_{0}(x)}{M_{2} \cdot \xi}\right)^{1 / 3} \in Q \\ & \text { else. }\end{cases}
$$

This is a convex function of $(\xi, z)$, as the set where it is finite is convex, and, by hypothesis, the function on such a set is also convex. Checking this is elementary but a bit tedious.

2. $v=u_{0}(x)$ : in this situation we have

$$
\varphi(x, u, \lambda, \xi, v, z)= \begin{cases}\min _{h \in\left[h_{-}, z\right] \cap Q} F(x, u, \lambda, \xi, h), & M_{2} \cdot \xi=0, \\ +\infty & \text { else. }\end{cases}
$$

This is again convex by our main structural assumption on $F$.

The proof of Theorem 1 is a direct consequence of Theorem 5 and Proposition 4 .

The generality of the cost functional permits us to associate with the state equation a huge class of optimization problems. We give some examples of such densities: the compliance case $F=f(x) u(x)$ or some other typical densities like $F=g(x) u(x), F=|\nabla u(x)|^{2}$, or the identification-type problem $F=\left|u(x)-u_{d}(x)\right|^{2}+$ $\left|\nabla u(x)-\nabla u_{d}(x)\right|^{2}$, where $u_{d} \in H^{1}(\Omega)$ is the observed deflection of the plate. Also, $F=F_{1}(x, u, \nabla u)$, where $F_{1}$ is continuous on the last two variables (but not necessarily 
convex) or $F=F_{2}\left(x, u, \nabla u, \nabla^{2} u\right)$, where $F_{2}$ is continuous on the last three variables and only convex in the $\nabla^{2} u$ variable, are densities for which the existence is ensured. Even with $F=F_{1}(x, u, \nabla u)+F_{3}(h)$ or $F=F_{2}\left(x, u, \nabla u, \nabla^{2} u\right)+F_{3}(h)$, where $F_{3}$ is any convex and nondecreasing function ( $F_{1}$ and $F_{2}$ as above), the existence of optimal classical minimizers is guaranteed.

3. The structure of the tensor $\boldsymbol{M}$. We have seen so far that the possibility of decomposing the tensor $M$ as the tensor product of two matrices is the crucial ingredient for having existence of optimal profiles. This will be so for special types of materials. In many sources from mechanics this is assumed as part of the model. See [2], [8]. Indeed, the equilibrium equation for the plate is often taken as

$$
D \Delta^{2} u=f,
$$

where the coefficient $D$ is the so-called flexural rigidity or the bending stiffness given by

$$
D=\frac{E h^{3}}{12\left(1-r^{2}\right)},
$$

where $h$ is the (constant) thickness of the plate, and $E$ and $r$ are, as before, Young's modulus and Poisson's ratio. When $h$ is nonconstant, then the equation of equilibrium must be written in the form

$$
\bar{D} \Delta\left(h^{3} \Delta\right)=f .
$$

This time

$$
\bar{D}=\frac{E}{12\left(1-r^{2}\right)} .
$$

Within this sort of model, the tensor $M$ is clearly decomposable with $M_{1}$ and $M_{2}$ multiples of the identity. In these cases, we can apply Theorem 1 to ensure the existence of optimal profiles.

The case of orthotropic materials is, however, very different. In fact, an orthotropic tensor is not decomposable.

Proposition 6. An orthotropic tensor is never decomposable.

The proof is elementary and well known to specialists. Indeed, by writing a fourth-order tensor as a $4 \times 4$ matrix in an organized way, we realize that the matrix corresponding to a orthotropic material is of the form

$$
\frac{4}{9} \frac{E^{4}}{\left(1-r^{2}\right)(1+r)^{2}}\left(\begin{array}{cccc}
1 & 0 & 0 & r \\
0 & 1 & 1 & 0 \\
0 & 1 & 1 & 0 \\
r & 0 & 0 & 1
\end{array}\right) .
$$

If such an $M$ were decomposable, this matrix would have to be a rank-one matrix, which is easily seen not to be the case.

4. Design with a nondecomposable tensor. We investigate in this section the same design problem for the plate under the assumption that the tensor of elastic constants is not decomposable so that Theorem 1 is not applicable. Indeed, it is well known, as indicated in the introduction, that in this situation nonexistence of an 
optimal profile may result, as the creation of highly oscillatory stiffeners may favor the overall rigidity of the plate.

As before, the goal is to choose the half-thickness $h$ and its corresponding deflection $u$, which minimizes an integral functional $L=L(h, u)$ given by

$$
L(h, u)=\int_{\Omega} F(x, u(x), \nabla u(x)) d x,
$$

where $F$ is assumed to be measurable in $x=\left(x_{1}, x_{2}\right)$ and continuous on the variables $u$ and $\nabla u$.

The class of materials is restricted by imposing an orthotropic condition, namely, the nonzero components of $M_{i j k l}$ are

$$
\begin{aligned}
& M_{1111}=M_{2222}=\frac{2}{3} \frac{E}{1-r^{2}}, \\
& M_{1122}=M_{2211}=\frac{2}{3} \frac{E r}{1-r^{2}}, \\
& M_{1212}=M_{1221}=M_{2112}=M_{2121}=\frac{E}{3(1+r)},
\end{aligned}
$$

where $r$ and $E$ stand for the Poisson ratio and the Young modulus, respectively. By our comments in the preceding section, this tensor is not decomposable.

We analyze this problem under the simplification that the thickness depends just on one variable $h(x)=h\left(x_{1}\right)$ for any $x_{1}$ in the interval

$$
(a, b) \doteq\left\{x_{1}: \text { there exists } x_{2} \in \mathbf{R} \text { such that }\left(x_{1}, x_{2}\right) \in \Omega\right\} .
$$

The design criterion is to minimize $L$ among all the plates whose half-thickness $h$ satisfies all the constraint indicated above. In other words, the aim is to solve

$$
\min _{h \in \mathcal{H}} L
$$

where

$$
\mathcal{H}=\left\{h \in L^{\infty}(a, b): h_{-} \leq h\left(x_{1}\right) \leq h_{+} \text {a.e. } x_{1} \in(a, b), \int_{\Omega} h\left(x_{1}\right) d x_{1} d x_{2} \leq V\right\} .
$$

Here $h_{-}, h_{+}$, and $V$ are as before.

As indicated before, it is widely recognized that the principle described in (12)(13) may have no solution. At least, Theorem 1 cannot be applied. This fact suggests performing a relaxation of the design problem to understand the nature of minimizing sequences of profiles. This entails defining a new admissibility set $\overline{\mathcal{H}}$ containing $\mathcal{H}$, and an extension $\bar{L}$ of $L$ such that

$$
\inf _{\mathcal{H}} L=\min _{\overline{\mathcal{H}}} \bar{L}
$$

It is interesting to notice that by introducing the relaxation $\min _{\overline{\mathcal{H}}} \bar{L}$, we are considering a problem whose solutions provide information about minimizing sequences of (12). However, it is important to look for the (full) relaxation, which introduces a minimal number of additional design variables. Ideally, just one more variable would 
be optimal. For the compliance functional, this was shown to be the case in [4] by making use of optimality conditions. We will prove that this is the case for many more cost functionals by revisiting some our our previous ideas on this problem [11].

The starting point for a relaxation is the lemma by Murat [12] and Tartar [18] related to H-convergence. It explains why the cubic-average and the harmonic cubicaverage play an important role in the relaxation for this problem. This lemma is only valid under our assumption of profiles depending only on $x_{1}$. The reader can consult [5] for a detailed proof of this result.

LEMma 7. Let $\left\{M^{(r)}\right\}$ be a sequence of orthotropic tensors bounded uniformly by $(d, D)$, i.e.,

$$
d|t|^{2} \leq \sum_{i, j, k, l} M_{i j k l}^{(r)} t_{i j} t_{k l}, \quad\left|\sum_{i j} M_{i j k l}^{(r)} t_{i j}\right| \leq D|t| \text { for every } k, l .
$$

Suppose that $M^{(r)}=M^{(r)}\left(x_{1}\right)$ and

$$
\begin{array}{rl}
\left(M_{1111}^{(r)}\right)^{-1} & *\left(M_{1111}^{(\infty)}\right)^{-1} \\
\left(M_{1122}^{(r)}\right)\left(M_{1111}^{(r)}\right)^{-1} & *\left(M_{1122}^{(\infty)}\right)\left(M_{1111}^{(\infty)}\right)^{-1}, \\
\left(M_{2222}^{(r)}\right)-\left(M_{1122}^{(r)}\right)^{2}\left(M_{1111}^{(r)}\right)^{-1} \stackrel{*}{\rightarrow}\left(M_{2222}^{(\infty)}\right)-\left(M_{1122}^{(\infty)}\right)^{2}\left(M_{1111}^{(\infty)}\right)^{-1}, \\
M_{1212}^{(r)} \stackrel{*}{\rightarrow} M_{1212}^{(\infty)} .
\end{array}
$$

If $u^{(r)}$ are the solutions of the equilibrium equation for the clamped plate with tensor $M^{(r)}$, and $u^{(\infty)}$ is the solution corresponding to $M^{(\infty)}$, then $u^{(r)}-u^{(\infty)}$ in $H_{0}^{2}(\Omega)$.

Because of the structure of the components of an orthotropic tensor, it is elementary to check that for a given sequence of designs $\left\{h_{j}\right\}$, if we define (in a unique way) the pair $(h, \theta)$ by putting

$$
\begin{gathered}
h_{j}^{3} \stackrel{*}{\rightarrow} \theta h_{+}^{3}+(1-\theta) h^{3}, \\
h_{j}^{-3} \stackrel{*}{\rightarrow} \theta h_{+}^{-3}+(1-\theta) h^{-3}
\end{gathered}
$$

for $\theta \in[0,1], h \in\left[h_{-}, h_{+}\right]$, and

$$
\begin{aligned}
& \bar{M}_{1111}=\frac{2}{3} c \frac{E}{\left(1-r^{2}\right)}, \\
& \bar{M}_{2222}=\frac{2}{3} m E+\frac{2}{3} c \frac{E r^{2}}{1-r^{2}}, \\
& \bar{M}_{1122}=\bar{M}_{2211}=\frac{2}{3} c \frac{E r}{1-r^{2}}, \\
& \bar{M}_{1212}=\bar{M}_{1221}=\bar{M}_{2112}=\bar{M}_{2121}=\frac{1}{3} m \frac{E}{(1+r)},
\end{aligned}
$$

where $m$ and $c$ denote the cubic average and the harmonic cubic-average of the pair $(\theta, h)$, respectively,

$$
\begin{aligned}
& m=\theta h_{+}^{3}+(1-\theta) h^{3}, \\
& c=\left(\theta h_{+}^{-3}+(1-\theta) h^{-3}\right)^{-1},
\end{aligned}
$$

then 


$$
L\left(h_{j}, u_{j}\right) \rightarrow \bar{L}(\theta, h)
$$

where

$$
\bar{L}(\theta, h)=\int_{\Omega} F(x, \bar{u}, \nabla \bar{u}) d x
$$

and $\bar{u}$ is the solution of the plate equation corresponding to the tensor $\bar{M}$. Notice that weak convergence in $H_{0}^{2}(\Omega)$ implies strong convergence in $H^{1}(\Omega)$.

This discussion suggests defining a relaxation as an optimization problem for pairs $(\theta, h)$ in

$$
\overline{\mathcal{H}}=\left\{(\theta, h): 0 \leq \theta \leq 1, h_{-} \leq h \leq h_{+}\right\}
$$

with cost

$$
\bar{L}(\theta, h)=\int_{\Omega} F(x, \bar{u}, \nabla \bar{u}) d x,
$$

where as above $\bar{u}$ is the solution of the equilibrium plate problem for tensor $\bar{M}$ obtained from $(\theta, h)$ through the cubic-average and the harmonic cubic-average as in (16) and (17).

This would essentially be the proof of Theorem 3 except for the fact that the parameter $V$ has not entered into our discussion. In fact, minimizing sequences of admissible designs must comply with

$$
\int_{\Omega} h_{j}(x) d x \leq V
$$

and we have not told how this parameter $V$ enters into the relaxation. How is $V$ to restrict further the pairs in $\overline{\mathcal{H}}$ ?

We observe that admissible pairs in $\overline{\mathcal{H}}$ come from the weak convergence of sequences $\left(h_{j}^{3}, h_{j}^{-3}\right)$. In order to relate $h_{j}$ to $\left(h_{j}^{3}, h_{j}^{-3}\right)$, we will look for a function $G$ so that

$$
h=G\left(h^{3}, h^{-3}\right), \quad h \in\left[h_{-}, h_{+}\right],
$$

and extend it by putting

$$
G\left(\theta h_{+}^{3}+(1-\theta) h^{3}, \theta h_{+}^{-3}+(1-\theta) h^{-3}\right)=\theta h_{+}+(1-\theta) h .
$$

If $G$ so defined turns out to be convex, then by the weak convergences in (15),

$$
\begin{aligned}
\lim _{j \rightarrow \infty} \int_{\Omega} h_{j}(x) d x & =\lim _{j \rightarrow \infty} \int_{\Omega} G\left(h_{j}^{3}(x), h_{j}^{-3}(x)\right) d x \\
& \geq \int_{\Omega} G\left(\theta(x) h_{+}^{3}+(1-\theta(x)) h(x)^{3}, \theta(x) h_{+}^{-3}+(1-\theta(x)) h(x)^{-3}\right) d x \\
& =\int_{\Omega}\left[\theta(x) h_{+}+(1-\theta(x)) h(x)\right] d x,
\end{aligned}
$$

so that we have

$$
\int_{\Omega}\left[\theta(x) h_{+}+(1-\theta(x)) h(x)\right] d x \leq V
$$


We then add this volume constraint to feasible pairs in $\overline{\mathcal{H}}$ :

$$
\overline{\mathcal{H}}=\left\{(\theta, h): 0 \leq \theta \leq 1, h_{-} \leq h \leq h_{+}, \int_{\Omega}\left[\theta(x) h_{+}+(1-\theta(x)) h(x)\right] d x \leq V\right\} .
$$

After the previous remarks, the full proof of Theorem 3 has been reduced to proving the convexity of the mapping $G$ described above. This convexity property for $G$ was proved in [11] (proof of Theorem 4.1). It is a nice, geometric argument, which we do not include here for the sake of brevity. It has nothing to do with the rest of the analysis in this work. One can also find in that paper how to recover admissible sequences of designs which are minimizing for the original problem from optimal pairs in $\overline{\mathcal{H}}$. This can be done in an elegant way by using Young measures associated with such sequences of designs.

\section{REFERENCES}

[1] N. Antonić And N. Balenović, Optimal design for plates and relaxation, in Proceedings of the 7th Annual International Conference in Operations Research (Rovinj, 1998), Math. Commun., 4 (1999), pp. 111-119.

[2] M. P. Bendsoe, Optimization of Structural Topology, Shape and Material, Springer, Berlin, 1995.

[3] E. Bonnetier and C. ConcA, Approximation of Young measures by functions and application to an optimal design problem for plates, Proc. Roy. Soc. Edinburgh Sect. A, 124 (1994), pp. 399-442.

[4] E. Bonnetier And C. Conca, Optimality conditions for a relaxed layout optimization problem, C. R. Acad. Sci. Paris Sér. I Math., 327 (1998), pp. 1005-1010.

[5] E. Bonnetier And M. Vogelius, Relaxation of a compliance functional for a plate optimization problem, in Application of Multiple Scaling in Mechanics, P. G. Ciarlet and E. Sánchez-Palencia, eds., Masson, Paris, 1987, pp. 31-53.

[6] K. T. Cheng and N. Olhoff, An investigation concerning optimal design of solid elastic plates, Internat. J. Solids Structures, 17 (1981), pp. 305-323.

[7] K. T. Cheng And N. Olhoff, Regularized formulation for optimal design of axisymmetric plates, Internat. J. Solids Structures, 18 (1982), pp. 153-169.

[8] D. Halim and S. O. Reza Moheimani, An optimization approach to optimal placement of collocated piezoelectric actuators and sensors on a thin plate, Mechatronics, 13 (2003), pp. $27-47$.

[9] R. V. Kohn And M. Vogelius, Thin plates with varying thickness, and their relation to structural optimization, in Homogenization and Effective Moduli of Materials and Media, IMA Vol. Math. Appl. 1, J. Ericksen, D. Kinderlehrer, R. Kohn, and J. L. Lions, eds., Springer, New York, 1986, pp. 126-149.

[10] X. Li and J. Yong, Optimal Control Theory for Infinite Dimensional Systems. Systems and Control: Foundations and Applications, Birkhäuser, Boston, 1995.

[11] J. Muñoz and P. Pedregal, On the relaxation of an optimal design problem for plates, Asymptot. Anal., 16 (1996), pp. 125-140.

[12] F. Murat, H-convergence, Séminaire d'analyse fonctionelle et numérique de l'Université d'Alger, 1977.

[13] P. Pedregal, On the generality of variational principles, Milan J. Math., 71 (2003), pp. 319356.

[14] P. Pedregal and A. Donoso, Optimal design of a plate of variable thickness: A variational approach in dimension one, Comput. Appl. Math., 22 (2003), pp. 75-89.

[15] T. RouBíč̆K, Maximum principle in optimal design of plates with stratified thickness, Appl. Math. Optim., 51 (2005), pp. 183-200.

[16] J. Sprekels And D. TiBA, A duality approach in the optimization of beams and plates, SIAM J. Control Optim., 37 (1998), pp. 486-501.

[17] J. Sprekels and D. Tiba, Optimization of clamped plates with discontinuous thickness, Systems Control Lett., 48 (2003), pp. 289-295.

[18] L. TaRTar, Cours Peccot, Collège de France, 1977. 